\title{
The GEF model: Assessment of fission-fragment properties over an extended region
}

\author{
Christelle Schmitt ${ }^{1, \star}$, Karl-Heinz Schmidt ${ }^{2, \star \star}$, and Beatriz Jurado ${ }^{2, \star \star \star}$ \\ ${ }^{1}$ IPHC, CNRS/IN2P3, 23 Rue du Loess, BP. 28, 67037 Strasbourg Cedex 2, France \\ ${ }^{2}$ CENBG, CNRS/IN2P3, Chemin du Solarium, BP. 120, 33175 Gradignan, France
}

\begin{abstract}
Calculations with the fission model GEF are performed over an extended region in fissioning-system mass $A$, charge $Z$, excitation energy $E^{*}$ and angular momentum $L$. The present contribution focuses on fission-fragment mass and isotopic distributions, benefiting from recent innovative measurements. The influence of the system $\left(A, Z, E^{*}\right.$, $L$ ) properties and of multi-chance fission is studied. The impact of accurate experimental knowledge about these properties and instrumental resolution is discussed. The need of as selective and accurate as possible experimental data to improve the model is demonstrated.
\end{abstract}

\section{Introduction}

In recent years, the need for codes characterized by high-predictive capabilities in anticipating consistently and simultaneously various fission observables has become more and more urgent for nuclear application purposes, but also for large-scale simulations in nuclear astrophysics as well as for guiding the development of purely microscopic theories. The General description of Fission observables (GEF) code [1] is a semi-empirical model responding to this demand. GEF is able to handle spontaneous and, more generally, induced fission of a compound nucleus formed in a nuclear reaction. The code gives access to a nearly-exhaustive set of fission observables, including fission probabilities, fragment properties, prompt and delayed neutrons and $\gamma$-rays. The present contribution focuses on fission-fragment mass and charge distributions. Recent innovative measurements are used to evaluate the accuracy of the GEF predictions for independently varied fissioning-system size, excitation energy and angular momentum, over a wide range beyond the region used in the model adjustement.

\section{The GEF model}

For fundamental research, assisting and interpreting measurements, and in various applications, fission models are essential. Models of different levels of sophistication have been developed over the years. Semi-classical models based on transport theory, as well as specific fully quantum mechanical frameworks, are currently encountering promising quantitative results (see e.g. Refs. [2,3] and

\footnotetext{
^e-mail: christelle.schmitt@iphc.cnrs.fr

$\star \star$ e-mail: schmidt-erzhausen@t-online.de

$\star \star \star$ e-mail: jurado@cenbg.in2p3.fr
} 
Ref. [4], respectively). Though, there is no unified model available yet and which can be used for any fissioning system. In addition, the corresponding codes do not implement a full description of the fission process, due to the many exit channels. Definitively, computing time is an issue, and still prevents use in large-scale calculations. Empirical and phenomenological models - again more or less sophisticated, were developed in parallel.

GEF is a semi-empirical model that exploits several general laws of mathematics and physics, combined with empirical information. In particular, GEF is based on the characteristics of the potential-energy landscape, some principles of quantum mechanics, nuclear dynamics, and statistical mechanics. Although GEF does not describe quantities from the microscopic level, it is not a mathematical fit of experimental observations. Also, it preserves the link between different fission observables. This theoretical framework defines the model in a qualitative way. The parameters entering into the formalism (around 100) are connected to the underlying physics; they specify the model in a quantitative way. These parameters were adjusted once with benchmark experimental data, and are taken the same for all systems over a large range of excitation energy. That has to be compared with the popular systematic of Ref. [5] which involve a similar number of parameters but for one fissioning system, only.

In praxis, GEF is a Monte-Carlo code which, starting from a specified, either entrance-channel reaction, or initial compound nucleus, computes the sequential decay of the system, including: possibility of pre-equilibrium emission, pre-scission evaporation (i.e., multi-chance fission) $)^{1},(A, Z)$ production of the primary (excited) fission fragments, post-scission evaporation and $\gamma$-ray emission, $(A$, $Z$ ) population of the final (secondary) products and their radioactive decay wherever suited by delayed neutrons and/or $\gamma$-rays. All along the calculation, the correlations between the different quantities are preserved. No parameter is to be adjusted. As mentioned above, these were fixed once, and are system-independent. The user is acting at two places: at the beginning, by specifying the reaction or initial compound nucleus, and at the end, by analyzing the output results. The latter can be provided upon request as an event-by-event list mode data file.

\subsection{Theoretical ideas and hypotheses}

A detailed presentation of the modeling of fission in GEF is reviewed in Ref. [1]. Those features which are crucial for the observables (primary and secondary fragment mass and charge) which this contribution focuses on are discussed briefly below.

\subsubsection{Fission barriers}

A first important aspect concerns the fission probability, which determines the initial properties of the nuclei that finally go to fission. In the calculation of this probability, the fission barrier plays a central role. In GEF, fission barriers are calculated within the idea of the macro-microscopic approach, and using of the topographic theorem [6]: The barrier is given by the sum of the macroscopic barrier (taken from the extended Thomas-Fermi prescription) and the additional binding energy by the empirical ground-state shell correction, plus enhanced pairing correlations at saddle. That implies that shell corrections at saddle are neglected, as suggested by the topographic theorem. The approach avoids the uncertainties of theoretical shell-correction energies and allows to discriminate different macroscopic models [7].

\footnotetext{
${ }^{1}$ In GEF, only pre-fission evaporation leads to multi-chance fission. Evaporation between saddle and scission occurs, when the nucleus is already "condemned" to fission.
} 


\subsubsection{Fission channels}

The second aspect of main importance in this work is the (primary) fragment $(A, Z)$ partition at scission. To model this, GEF exploits the basic ideas of the early manifestation of fragment shells [8], the concept of quantum oscillators for the fission modes, and dynamical freezing [11]. In this frame, the fission channels are related to the statistical population of quantum oscillators in the mass-asymmetry degree of freedom that form the fission valleys. The quantum oscillator of each channel is characterized by three parameters (position, depth, and curvature) that are traced back to the macroscopic potential and to shells in the proton and neutron subsystems of both fragments, which are assumed to be effective already little beyond the outer saddle [8]. These shells are assumed to be the same for all fissioning systems. It is the superposition of different shells and the interaction with the macroscopic potential that create the mass distributions which differ for different systems [9]. Note that these shells also determine the shape (mainly quadrupole deformation) of the nascent fragments at scission. According to Strutinsky-type calculations, the fragment shapes are found to be a linearly increasing function of proton, respectively neutron, number in regions between closed spherical shells [10]. Also the charge-polarization (deviation of the $N / Z$ degree of freedom at scission - mean value and fluctuations - from the "UCD" value of the fissioning nucleus) is treated by the corresponding quantum oscillator [11].

Adjustement of the predictions by the above formalism to benchmark experimental mass and charge distributions over a wide region of the nuclear chart showed that four fission channels are necessary: the symmetric SL channel, and three asymmetric channels ("standard" S1 and S2, and very asymmetric SA). This empirical adjustement procedure has fixed the value of all parameters of the model.

\subsubsection{Energy sorting}

The third aspect which is important for this contribution concerns fission energetics, and in particular energy sharing between the fragments at scission. It determines the decay of the primary, and population of the secondary, fragments. By the influence of pairing correlations, the nuclear temperature below the critical pairing energy is assumed to be constant [12]. Therefore, the di-nuclear system between saddle and scission consists of two coupled microscopic thermostates [13]. This leads to a sorting process of the available intrinsic energy and of unpaired nucleons before scission [14-16].

\section{Results}

Within the aforementioned framework, the GEF code succeeded to describe a large spectrum of observables [1] over a wide range of nuclides, excitation energy and angular momentum. The simplicity and efficiency in handling and running GEF makes this code used by the community for various purposes including the treatment of experimental data, e.g. Ref. [17], guidance for theories, e.g. Ref. [18], interpretation of data, e.g. Refs. [19, 20]. Implementation in large-scale calculations at Radioactive Beam Facilities and in astrophysics (e.g. Refs. [21, 22]) has been done. Finally, the GEF code can be used for examining the consistency of experimental results and for evaluation [1]. For a more comprehensive validation, and possible improvement, of the GEF code, new and high-quality experimental data are mandatory [23]. A step in this direction is proposed in this contribution benefiting from recent innovative measurements. 


\subsection{Dependence of fragment mass partition on the fissioning system properties}

Using multi-nucleon transfer-induced-fission from ${ }^{18} \mathrm{O}+{ }^{232} \mathrm{Th}$ collisions, Leguillon et al. [24] measured in a single experiment the fragment pre-neutron mass distribution for about 15 fissioning isotopes between ${ }^{231} \mathrm{Th}$ and ${ }^{238} \mathrm{U}$, over a wide excitation energy range, from threshold to about $60 \mathrm{MeV}$. The data set of Ref. [24] is particularly relevant for the present concern. First, it permits to probe the ability of GEF to describe the evolution with fissioning $N$ and $Z$ in a region not much explored yet, and, importantly, well beyond the region of the model adjustement. Second, the $E^{*}$ domain covered in Ref. [24] is a critical one: $i$ ) multi-chance fission progressively sets in, and $i i$ ) shell effects progressively vanish. Finally, it shall be emphasized that all systems are produced, measured, and analyzed in the same way, what ensures consistency between the results obtained for the different fissioning nuclei.

The comparison of experiment and GEF calculation is displayed in Fig. 1. Note that, in experiment, due to limited resolution and statistics, the initial excitation energy is distributed over domains which are $10 \mathrm{MeV}$-wide about the value given in the figure. The calculation was done for a single $E^{*}$ (quoted in the figure), which was taken as the central value of the experimental window. The survey of the chart of Fig. 1 shows that GEF describes rather well the evolution of the distribution, with initial system $N$ and $Z$, and $E^{*}$. A detailed inspection, though, shows some deviation. The latter is attributed to several reasons. Transfer reactions can induce a non-negligible amount of angular momentum $L$ in the fissioning compound nucleus, what affects the fragment mass distribution; in the calculation of Fig. 1, $L$ was set to zero. Another aspect is the $10 \mathrm{MeV}$-wide $E^{*}$ interval inherent to the experiment, and which influences the mass distribution also; the calculation assumed a well-defined $E^{*}$ situated mid-way in this interval. Another effect (purely experimental) is the limited mass resolution $(\sigma=$ 6 units quoted in [24]) which is not taken into account in the calculated curves of Fig. 1. Last, but not least, model deficiencies cannot be excluded.

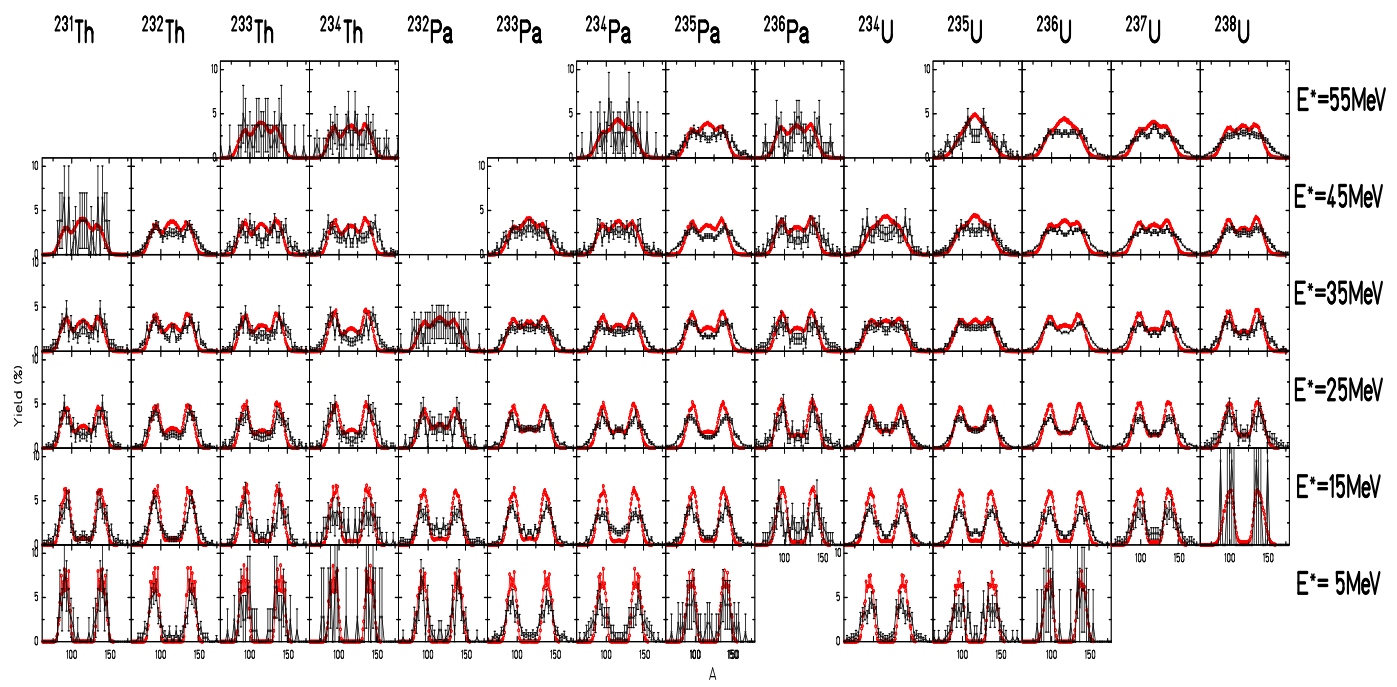

Figure 1. Fission-fragment pre-neutron mass distribution for various systems as measured in Ref. [24] (black) and compared with GEF calculations (red). Initial compound nucleus isotopes are ordered by columns, and initial mean $E^{*}$ values are ordered by rows.

To investigate the magnitude of the aforementioned reasons of deviation between the measurement and GEF, the calculations were repeated at various $L$ and $E^{*}$ values. Also, they were folded with 
the experimental resolution. A sample of results is gathered in Fig. 2. The GEF model suggests that the influence of angular momentum is present, but not that strong for the present systems. The width of the $E^{*}$ window can have a large impact. Experimental mass resolution has a non-negligible influence. Finally, the crucial need to account for multi-chance fission is also illustrated in Fig. 2, even at the "medium" $E^{*}$ covered here. In conclusion, neglecting these four aspects can yield an un-proper comparison of experiment and theory, and may even lead to data mis-interpretation.
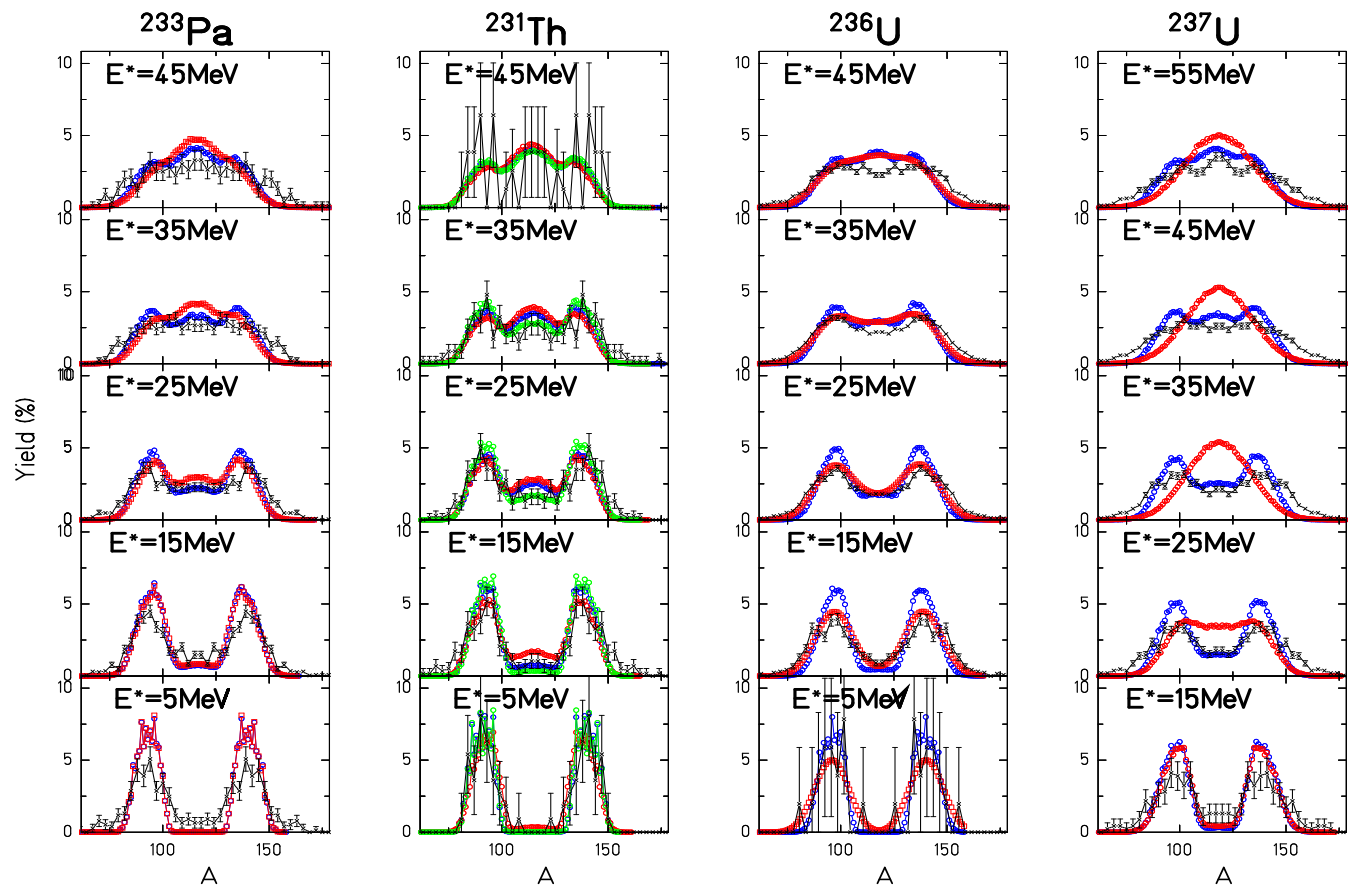

Figure 2. Fission-fragment pre-neutron mass distribution for selected systems from Ref. [24] (black) and compared with various GEF calculations. First column: calculations with $L=0$ (blue) and $L=20$ (red) as a typical maximum value in transfer; second column: calculations with $E^{*}$ taken as the center $\left.\left(<E^{*}\right\rangle\right)$ (blue) of the experimental interval, and as the lower $\left(<E^{*}>-5 \mathrm{MeV}\right)$ (red) and upper $\left.\left(<E^{*}\right\rangle+5 \mathrm{MeV}\right)$ (green) limit; third column: calculations assuming unique mass resolution (blue) and folded with the experimental resolution of $\sigma=6$ units (red); fourth column: calculations without (red) and with (blue) multi-chance fission taken into account.

The potential of GEF for unfolding experimental observations from the aforementioned effects is demonstrated in Fig. 3. The mass distribution measured at Tokai [24] for one example is compared to a measurement performed in Jyvaskyla [25] for the same initial system. There, fission was induced by bombarding a heavy target with a proton beam. In such a configuration, the angular momentum imparted to the system is small, and the excitation energy is well defined. In addition, the set up of Ref. [25] permitted to achieve a mass resolution of $\sigma$ around 1.5 unit. Also, the statistics collected was high. Unfortunately, according to the method, only one system could be measured. The deviation between the Tokai and Jyvaskyla measurements and the good agreement between GEF and the Jyvaskyla data set corroborate the non-negligible intricate interplay of $L, E^{*}$ and resolution effects. Furthermore, it demonstrates that the GEF code is a relevant tool to trace back the different influences, and possibly unfold each of them in the measurement. In the present specific case, Fig. 2 
would suggest that most of the discrepancy observed between the two experiments in Fig. 3 may be ascribed to resolution effects.

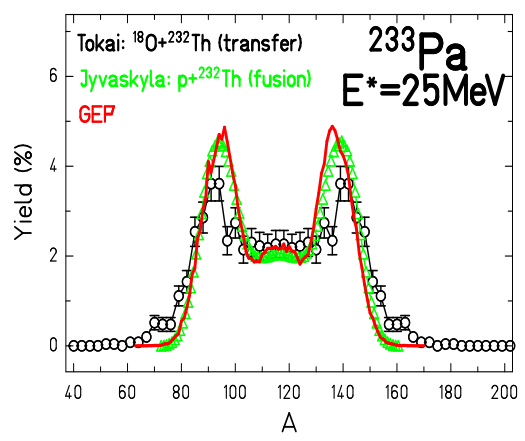

Figure 3. Fission-fragment pre-neutron mass distribution for ${ }^{233} \mathrm{~Pa}$ at $E^{*}=25 \mathrm{MeV}$ as measured at Tokai (black) and at Jyvaskyla (green) in transfer- and proton-induced fission, respectively. The GEF calculation (red) with $L=$ 0 and $E^{*}=25 \mathrm{MeV}$ overlaps reasonably well with the distribution of Jyvaskyla. This experiment was performed under better-defined conditions, as inherent to the method. See also text.

The chart measured at Tokai, Fig. 1, allows to study the evolution with mass, charge, excitation energy, and possibly angular momentum, of the fissioning system. Yet, Figs. 1 to 3 also show that to probe the influence of $E^{*}$ and $L$ in detail the data set of Ref. [24] is still lacking experimental accuracy (in terms of fissioning system properties and fragment mass) and statistics.

\subsection{Fragment isotopic distribution}

In parallel to the above study scanning the $\left(N, Z, E^{*}, L\right)$ fissioning hyperspace, the validation of GEF can still be performed on another front, thanks to the emergence of recent experiments at VAMOS/GANIL and SOFIA/GSI. These gave access for the first time to the complete nuclide production in low excitation-energy fission for several actinides. Mass and charge identification was achieved with unique precision for all the light and heavy fragments. Such precise and complete isotopic distributions certainly constitute a very valuable constraint for any model of fission. Yet, most models - among very sophisticated ones, restrict to either mass or charge partitioning. That is, a fixed ratio (usually the one of the compound system) is assumed between $N$ and $Z$. In GEF, fragment $N$ and $Z$ are two independent degrees of freedom, and whose population is governed by the physics outlined in Sect. 2.

The approach at VAMOS/GANIL has some similarity with the one used at Tokai. Namely, fission is induced by transfer reactions around the Coulomb barrier with ${ }^{238} \mathrm{U}(6.1 \mathrm{MeV} /$ nucleon $)+{ }^{12} \mathrm{C}$ collisions, and the initial compound-nucleus mass, charge and excitation energy is deduced eventby-event from the measurement. As compared to Ref. [24], inverse kinematics is employed, and use of the VAMOS magnetic spectrometer permits to intercept and isotopically identify one of the fragments [26]. Unfortunately, statistical significance is available for a few systems only, between ${ }^{238} \mathrm{U}$ and ${ }^{250} \mathrm{Cf}$. According to the detection method, post-neutron masses are measured at GANIL. In Ref. [27, 28] good agreement was observed between the experimental and GEF-predicted mass and charge integral distributions. A deeper survey is here proposed [29] by comparing the experimental and calculated isotopic distributions for the initial ${ }^{240} \mathrm{Pu}$ compound system excited at about $10 \mathrm{MeV}$ (FWHM $\approx 7 \mathrm{MeV}$ ), see Fig. 4 . The description by GEF is observed to be reasonable, over the entire 


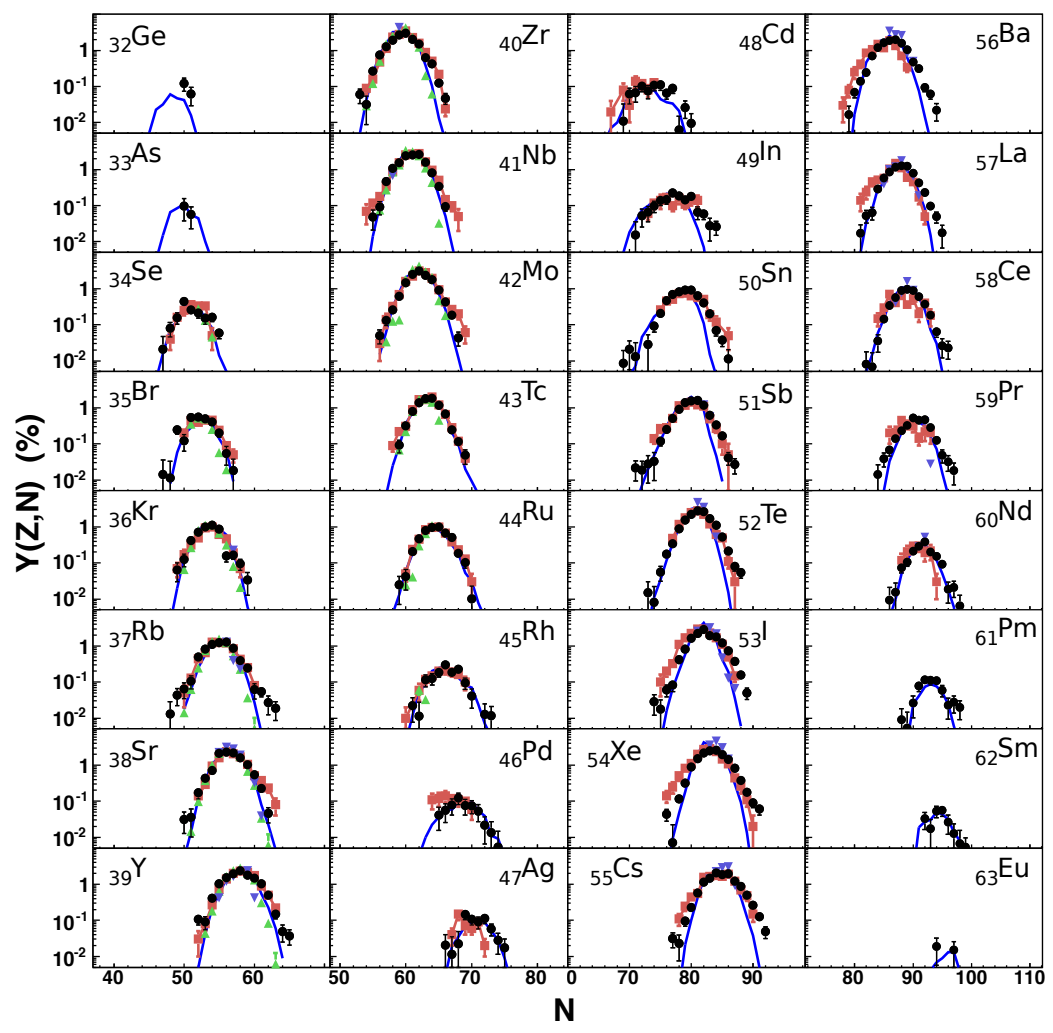

Figure 4. Fragment post-neutron $N$ distribution for elements between $\mathrm{Ge}$ and Eu as measured at VAMOS (black and red) in low-energy fission of ${ }^{240} \mathrm{Pu}$. Experiment is compared with GEF (blue). Figure is adapted from Ref. [29].

range from light to heavy fragments. Some deviation is visible at the edges of the distribution for a few elements. This is attributed so far to still not perfect $Z$ identification in the experiment [30].

The approach at SOFIA/GSI is based on fission induced by electromagnetic excitation following heavy-ion collisions at relativistic energy [31]. To make available, in a single experiment, a wide variety of fissioning isotopes, a two-step scheme is employed. The reaction ${ }^{238} \mathrm{U}(1000 \mathrm{MeV} /$ nucleon $)$ $+{ }^{9} \mathrm{Be}$ is used to produce by fragmentation a large set of stable and $\mathrm{r}$ adioactive products. These are used as secondary relativistic beams which bombard a heavy $\left({ }^{238} \mathrm{U}\right.$ or $\left.{ }^{208} \mathrm{~Pb}\right)$ secondary target, what induces fission of the secondary beam by electromagnetic excitation with an average $E^{*} \approx 14 \mathrm{MeV}$ (extending from threshold up to $35 \mathrm{MeV}$ ). As compared to the GANIL run, inverse kinematics is employed at SOFIA also, but the set up gives access to the post-scission mass and charge, with unique precision, for both fragments in coincidence. However, the initial excitation energy is not measured event-by-event, yielding the issue of an intricate convolution of a complex $E^{*}$ distribution [20]. Few systems were already published, among which the results for low-energy fission of ${ }^{235,238} \mathrm{U}$. The experimental and calculated isotopic distributions are reported in Fig. 5 for the case of ${ }^{238} U$ [20]. Overall the model describes the measurement well. It is noteworthy to emphasize that the isotopic distribution is seen to deviate from a Gaussian shape for many elements, and that the calculation is capable of explaining this observation [20]. Though, discrepancy between experiment and theory 


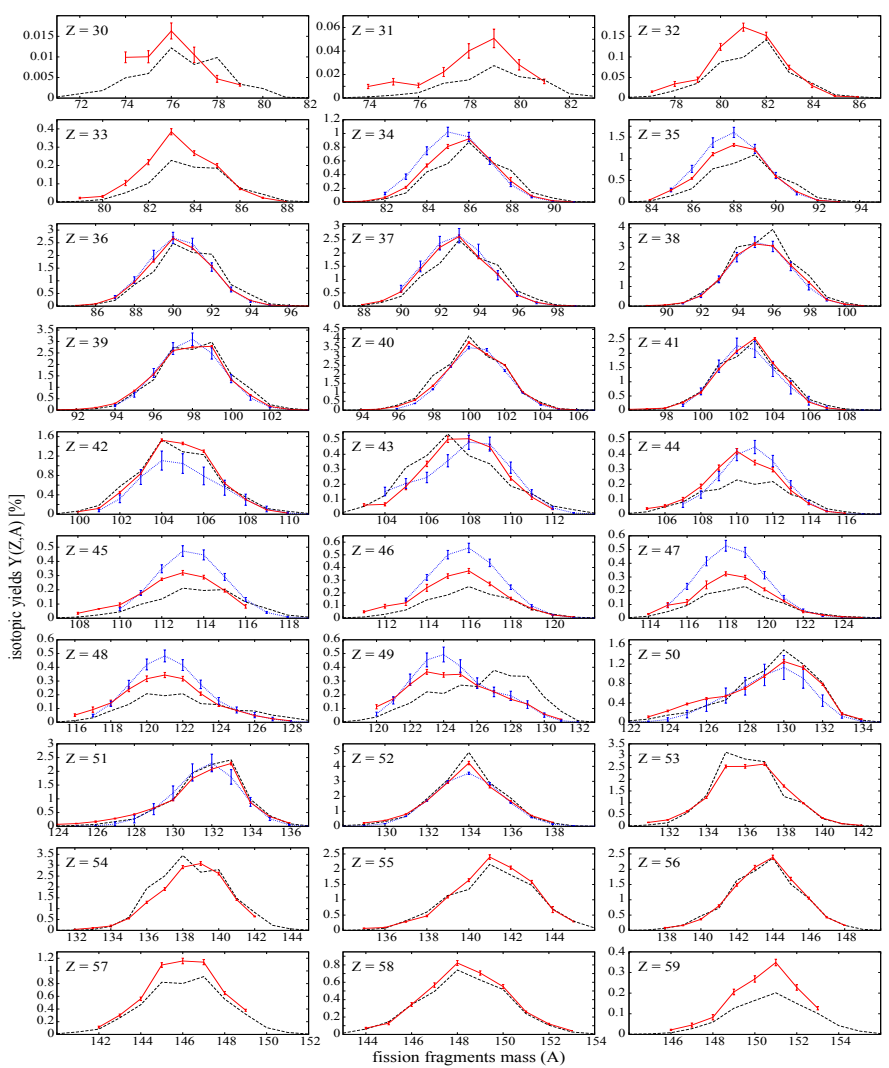

Figure 5. Fragment post-neutron mass distribution for elements between Ga and Pr as measured at SOFIA (red) and earlier at GSI (blue) in low-energy fission of ${ }^{238} \mathrm{U}$. Experiment is compared with GEF (black). Figure is adapted from Ref. [20].

exists in the symmetric fission region, and whose origin is under investigation. Note that this is the region where the yields are smallest, and where accurate account of $E^{*}$ may be critical. A slight underestimation by GEF of the yields around symmetry with increasing excitation energy was already seen for selected cases in Refs. [1,32].

According to Figs. 4 and 5, and within the experimental control of $E^{*}$, GEF describes consistently the fragment $(N, Z)$ population. Gaining further insight calls for more exclusive experimental information (in terms of e.g. $E^{*}$ and $L$ ).

\subsection{Neutron multiplicity}

According to the direct identification of both fragments in mass, the SOFIA experiment gives also access to the total neutron multiplicity $v_{t o t}$ for each $(N, Z)$ fission partition. This multiplicity is reported in Fig. 6 for electromagnetic-induced fission of ${ }^{235} \mathrm{U}$. The calculation (left) is in agreement with the measurement (right). That could already be inferred from Fig. 5 where the experimental and calculated peak localizations coincide well. Comparing the measured and predicted $v_{t o t}$ quantity can be used to study fission energetics. Though, to get deeper insight, and namely investigate the debated 
issue of energy sharing at scission, requires to consider the neutron multiplicity $v_{L, H}$ per fragment. That is not available from experiment on the $(N, Z)$ plane yet.

The clear dependence of $v_{t o t}$ on $N$ and $Z$ seen in Fig. 6 implies that accurate account of postscission evaporation in data analysis aiming at recovering primary fragment masses from measured secondary ones requires to perform a correction that depends on both fragment $N$ and $Z$, at the risk of data mis-interpretation. So far, due to lack of experimental information in the two-dimensional plane, neutron corrections are usually done as function of fragment mass, only (see e.g. Ref. [17] and therein).
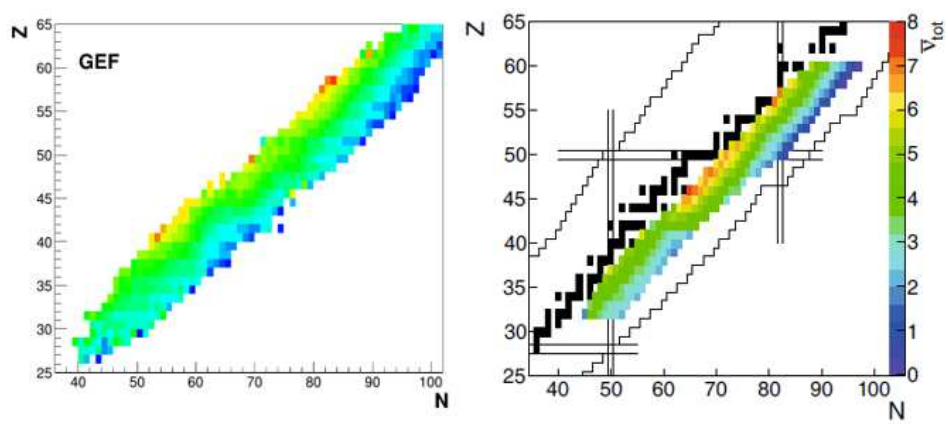

Figure 6. Right: total neutron multiplicity $v_{t o t}$ as function of fragment post-neutron $(N, Z)$ partition as deduced at SOFIA for low-energy fission of ${ }^{235} \mathrm{U}$. Adapted from Ref. [31]. Left: identical to the right but for the GEF calculation.

\subsection{Importance of correlations}

As mentioned in Sect. 2, GEF provides the link between the different fission quantities. That is, it preserves the correlations between these quantities. Calculated correlations can be fruitfully used in interpreting experimental observations, since the inclusive measurements required to extract correlations are rare. As a demonstration of the potential of GEF in this respect, let us consider again the isotopic distributions measured at SOFIA and shown in Fig. 5. The experimental (red) distribution for $\operatorname{Sn}(Z=50)$ has a main peak at $A \approx 130$ and exhibits a pronounced shoulder centered at $A \approx 126$. That was interpreted in Ref. [20] as being due to the contribution of both the SL and S1 fssion modes to the production of this element (see also Fig. 22 of Ref. [20]). A left tail is seen in the GEF calculation, too, although less pronounced than in experiment. In order to corroborate the experimental interpretation, knowledge on, either total kinetic energy (TKE), or the aforementioned $v_{L, H}$, is best suited. This, because the SL and S1 components are associated to higher and lower intrinsic excitation energy at scission, respectively, i.e., lower/larger (higher/smaller) values for $T K E / v_{L, H}$. Since these observables are not available yet from SOFIA, support from model calculations is welcome. It is found (not shown) that GEF expects a contribution from SL and S1 to the Sn isotopic distribution, with distinct $T K E$ and $v_{L, H}$.

\section{Conclusions}

Comparison of recent innovative measurements with calculations by GEF shows the capability of the code to describe reasonably the experimental data over a wide range of fissioning system mass, 
charge, excitation energy and angular momentum. In addition, the GEF results may help in the discrimination between excitation energy, angular momentum and experimental resolution effects. This study highlights the further need of high-quality experimental information for a better understanding of the fission process.

\section{References}

[1] K.-H. Schmidt, B. Jurado, Ch. Amouroux, and C. Schmitt, Nucl. Data Sheets 131, 107 (2016)

[2] J. Randrup and P. Möller, Phys. Rev. C 88, 064606 (2013)

[3] M.D. Usang et al., Phys. Rev. C 94, 044602 (2016)

[4] D. Regnier, N. Dubray, N. Schunck, and M. Verriere, Phys. Rev. C 93, 054611 (2016)

[5] A.C. Wahl, Report LA-13928 (Los Alamos National Laboratory, 2002) (unpublished)

[6] W.D. Myers and W.J. Swiatecki, Nucl. Phys. A 601, 14 (1996)

[7] A. Kelić and K.-H. Schmidt, Phys. Lett. B 634, 362 (2006)

[8] U. Mosel and H.W. Schmitt, Nucl. Phys. A 165, 73 (1971)

[9] K.-H. Schmidt, A. Kelić, and M.V. Ricciardi, Europh. Lett. 83, 32001 (2008)

[10] B.D. Wilkins, E.P. Steinberg, and R.R. Chasman, Phys. Rev. C 14, 1832 (1976)

[11] H. Nifenecker, J. Physique Lett. 41, 47 (1980)

[12] K.-H. Schmidt and B. Jurado, Phys. Rev. C 86, 044322 (2012)

[13] K.-H. Schmidt and B. Jurado, Phys. Rev. Lett. 104, 212501 (2010)

[14] K.-H. Schmidt and B. Jurado, Phys. Rev. C 83, 014607 (2011)

[15] K.-H. Schmidt and B. Jurado, Phys. Rev. C 83, 061601 (2011)

[16] B. Jurado and K.-H. Schmidt, J. Phys. G: Nucl. Part. Phys. 42, 055101 (2015)

[17] D. Duke et al., Phys. Rev. C 94, 054604 (2016)

[18] W. Younes, Eur. Phys. J. A 53, 109 (2017)

[19] J. Khuyagbaatar, private communication

[20] E. Pellereau et al., Phys. Rev. C 95, 054603 (2017)

[21] A. Al-Adili et al., Eur. Phys. J. A 51, 59 (2015)

[22] S. Goriely et al., Eur. Phys. J. A 51, 22 (2015)

[23] K.-H. Schmidt, B. Jurado, C. Schmitt, Contribution to the NEA Nuclear Data Week - JEFF Meetings, OECD Nuclear Energy Agency, Paris, France (2017) (unpublished)

[24] R. Leguillon et al., Phys. Lett. B 761, 125 (2016)

[25] E.M. Kozulin et al., FUSION06 Conference, Venezia, Italy (2006) (unpublished)

[26] M. Caamano et al., Phys. Rev. C 88, 024605 (2013)

[27] M. Caamano et al., Phys. Rev. C 92, 034606 (2015)

[28] D. Ramos et al., Acta Pol. B 46, 329 (2015)

[29] D. Ramos, PhD, University of Santiago de Compostela (2016) (unpublished)

[30] F. Farget, private communication

[31] J.-F. Martin et al., Eur. Phys. J. A 51, 174 (2015)

[32] J.N. Wilson et al., Phys. Rev. Lett. 118, 222501 (2017) 